\title{
Questes
}

Revue pluridisciplinaire d'études médiévales

\section{Copie, authenticité et originalité dans les chansonniers de trouvères : un bref panorama}

\section{Georges Veyssière}

\section{OpenEdition}

\section{Journals}

Édition électronique

URL : http://journals.openedition.org/questes/3541

DOI : 10.4000/questes.3541

ISSN : 2109-9472

\section{Éditeur}

Les Amis de Questes

\section{Édition imprimée}

Date de publication : 15 janvier 2015

Pagination : 87-94

ISSN : 2102-7188

\section{Référence électronique}

Georges Veyssière, «Copie, authenticité et originalité dans les chansonniers de trouvères : un bref panorama », Questes [En ligne], 29 | 2015, mis en ligne le 20 juin 2015, consulté le 30 avril 2019. URL http://journals.openedition.org/questes/3541; DOI : 10.4000/questes.3541 


\title{
Copie, authenticité et originalité dans les chansonniers de trouvères : un bref panorama
}

\author{
Georges VEYSSIÈRE \\ Université Paris-Sorbonne (Paris IV)
}

Dans le domaine de la lyrique des trouvères, d'après l'ouvrage bibliographique le plus récent relatif à ce domaine ${ }^{1}$, on peut estimer qu'il existe plus de deux mille chansons et une trentaine de manuscrits que l'on a coutume d'appeler chansonniers. Contrairement aux chansonniers occitans qui sont aujourd'hui plutôt bien connus, ils se distinguent notamment par l'absence de vidas - biographies où la fiction se mêle aisément à la vérité historique - et de razos - circonstances imaginaires dans lesquelles les chansons ont été composées. Cependant, cette distinction est adoptée ici uniquement pour sa commodité, car bien des manuscrits se signalent par la coexistence de chansons en oc et en oï ${ }^{2}$.

Le présent article tentera donc de souligner la multiplicité de

\footnotetext{
${ }^{1}$ Robert White Linker, A Bibliography of old French lyrics, Université du Mississippi, coll. «Romance Monographs », 31, 1979. Pour les manuscrits, les sigles utilisés aujourd'hui par la majorité des chercheurs, y compris par l'auteur de ces lignes, sont ceux d'Eduard Schwan, Die Altfranzösischen Liederhandschriften, ihr Verhältniss, ihre Entstehung und ihre Bestimmung. Eine litterarhistorische Untersuchung, Berlin, Weidmann, 1886. Ils ont remplacé ceux de Gaston Raynaud, Bibliographie des chansonniers français des XIII et XIV siècles, Paris, F. Vieweg, 1884, 2 tomes. Les chansons sont habituellement numérotées en suivant le système de Raynaud-Spanke (abréviation « $\mathrm{R} »$ ou « RS ») qui adopte un classement alphabétique en fonction des rimes) et celui de Linker (classement alphabétique par noms de trouvères).

${ }^{2}$ On songe notamment au caractère composite du chansonnier de Modène, Biblioteca Nazionale Estense, Estero 45, Alpha R. 4.4. Voir notamment l'article de Fabio Zinelli, «D'une collection de tables de chansonniers romans (avec quelques remarques sur le chansonnier Estense) », Romania, 122, 2004, p. 46-110.
} 
phénomènes que peut recouvrir le terme chansonnier et soulèvera quelques problèmes qui se posent aux éditeurs de textes lyriques.

\section{Chansonnier, un terme unique pour une réalité plurielle}

L'appellation de chansonniers peut donner l'impression qu'il s'agit de recueils constitués exclusivement de pièces lyriques, impression renforcée par le système de sigles. Si le manuscrit O (Paris, BnF, fr. 846), datant de la fin du XIII ${ }^{\mathrm{e}}$ siècle et qui recense plus de 350 chansons, peut être qualifié de chansonnier au sens strict, il arrive bien souvent que ces recueils contiennent plusieurs éléments génériques différents ${ }^{3}$. Le chansonnier $\mathrm{V}$ (Paris, BnF, fr. 24406) en est un bon exemple. Le recueil est composé luimême de deux manuscrits, le premier regroupant 300 chansons dépourvues de rubriques mentionnant les noms des trouvères, mais classées par auteur $\left(f^{\circ} 1 r^{0}-119 v^{\circ}\right)$, et dont l'écriture pourrait dater de la fin du XIII siècle, tandis que le deuxième manuscrit, révélant une autre main, remonte au début du $\mathrm{XIV}^{\mathrm{e}}$ siècle et comprend un Traité des quatre nécessaires $\left(\mathrm{f}^{\mathrm{0}} 120 \mathrm{r}^{\mathrm{o}}-140 \mathrm{r}^{\mathrm{o}}\right)$, dont l'auteur ne nous est pas connu, et le Bestiaire d'amour de Richart de Fournival $\left(\mathrm{f}^{\mathrm{o}} 141 \mathrm{r}^{\mathrm{o}}-148 \mathrm{r}^{\circ}\right)$, suivis d'une trentaine de chansons dédiées à la Vierge ( $\left.\mathrm{f}^{\mathrm{0}} 148 \mathrm{r}^{\circ}-155\right)$. De même, dans le cas de P (Paris, BnF, fr. 847), manuscrit de 228 folios composé par plusieurs mains vers la fin du $\mathrm{XIII}^{\mathrm{e}}$ siècle, une section initiale d'environ 300 chansons (jusqu'au $\mathrm{f}^{\mathrm{o}} 203 \mathrm{v}^{\mathrm{o}}$ ) est suivie du Roman du Verger de l'Arbre d'Amour $\left(\mathrm{f}^{\mathrm{o}} 204 \mathrm{r}^{\circ}-210 \mathrm{v}^{\circ}\right)$, poème allégorique, avant de se clore par une trentaine de chansons d'Adam de la Halle $\left(f^{0} 211 r^{0}-228 v^{0}\right)$.

\footnotetext{
${ }^{3}$ La nécessité d'une distinction rigoureuse entre chansonniers au sens strict et chansonniers au sens large a été déjà rappelée par Maria Carla Battelli, "Les manuscrits et le texte : typologie des recueils lyriques en ancien français », Revue des langues romanes, 100, 1996, p. 111-129. Pour une typologie encore plus développée des manuscrits de trouvères, voir du même auteur, « Le antologie poetiche in anticofrancese », Critica del testo, II / 1, 1999, p. 141-180.
} 
Les chansonniers se distinguent également les uns des autres par la façon dont les chansons sont ordonnées et classées au sein du manuscrit. Ainsi, dans $\mathrm{O}$, les chansons semblent rangées alphabétiquement en plusieurs groupes en fonction de la première lettre de leur incipit, ce qui est une pratique observée généralement dans les manuscrits de motets. En revanche, l'ordre des chansons dans chacun de ces groupes est loin d'être rigoureux. Pour ne prendre que l'exemple de la section consacrée à la lettre « A », le lecteur du chansonnier trouve au tout premier folio la célèbre chanson de Thibaut de Champagne « Ausi cum l'unicorne sui...» (RS2075, L240-3) juste avant «Amours me fait comencier... » $(\mathrm{RS} 1268, \mathrm{~L} 240-2) \mathrm{du}$ même trouvère, puis « $\mathrm{A}$ enviz senc mal qui ne l'a apris... » (RS1521, L240-1), alors que ces chansons auraient dû être dans le sens inverse si l'ordre alphabétique avait été scrupuleusement respecté. Dans les autres parties du chansonnier, des regroupements par auteurs sont également observables au sein de ces sections, voire des doublons, comme le cas d'une chanson du Châtelain de Coucy aux folios $74 r^{\circ}$ et $78 v^{\circ}$ (RS1009, L38-8), ce qui tendrait à montrer que le copiste disposait de plusieurs manuscrits.

C'est le critère du genre des chansons qui intervient dans la structuration du manuscrit I (Oxford, Bodleian Library, Douce 308), et ce dans l'ordre suivant: «grands chants » (section qui regroupe les chansons d'amour), « estampies », « jeux-partis », « pastourelles », « ballettes », «sottes chansons» et «motets», cette dernière section n'étant pas indiquée dans l'index du manuscrit.

L'ordre des chansons peut enfin dépendre du statut social des trouvères, comme le montre le chansonnier M (Paris, BnF, fr. 844$)^{4}$, où l'on distingue trois groupes: un premier constitué de seigneurs, que les miniatures représentent à cheval, munis d'un écu; un deuxième groupe

\footnotetext{
${ }^{4}$ Chansonnier $\mathrm{W}$ pour les troubadours.
} 
composé de « maistres » (clercs) comme Guillaume le Vinier ; un troisième comprenant des trouvères comme Pierequin de la Coupelle ${ }^{5}$. Ce chansonnier se distingue également par la présence d'un petit chansonnier indépendant qui regroupe exclusivement des chansons de Thibaut de Champagne ( $\mathrm{f}^{\mathrm{o}} 13$, puis $59-77$, que l'on remarque clairement par l'absence de rubriques et par l'usage d'une encre plus claire), appelé $\mathrm{t}$ depuis Schwan ${ }^{6}$. On a pu se demander si ce chansonnier indépendant (Liederbuch) était celui évoqué dans les Grandes Chroniques de France :

Si fist entre luy et Gace Brulé les plus belles chansons et les plus délitables et mélodieuses qui oncques fussent oïes en chançon ne en vielle. Et les fist escrire en sa sale a Provins et en celle de Troyes, et sont appellées Les Chançons au Roy de Navarre ${ }^{7}$.

Si l'on ne peut pas admettre aveuglément une telle affirmation, il apparaît de fait qu'un certain nombre de chansons de Thibaut se trouvent dans un ordre globalement similaire dans plusieurs manuscrits, notamment dans $\mathrm{T}$ (Paris, BnF, fr. 12615). Dans N (Paris, BnF, fr. 845), K (Paris, BnF, Arsenal, ms. 5198) et $\mathrm{V}$, la section $\mathrm{t}$ suit souvent le même ordre, pour former une petite anthologie et un échantillon représentatif des compositions de Thibaut de Champagne ${ }^{8}$ : une chanson inaugurale

\footnotetext{
${ }^{5}$ La miniature représentant Pierequin de la Coupelle au $\mathrm{f}^{\mathrm{o}} 163 \mathrm{r}^{\mathrm{o}}$ pourrait induire en erreur le lecteur du manuscrit, car on y distingue une couronne qui est purement symbolique et rappelle que Pierequin a été couronné lors d'un "puy », compétition poétique qui a régulièrement eu lieu dans la ville d'Arras tout au long du XIII ${ }^{\mathrm{e}}$ siècle.

${ }^{6}$ Cf. Eduard Schwan, op. cit., p. 223-230 et 270-273. Sur le chansonnier M, voir Maria Carla Battelli, «Il codice Parigi, Bibl. nat. F. fr. 844 : un canzoniere disordinato ?», dans La Filologia romanza e i codici. Atti del Convegno, Messina, Università degli Studi - Facoltà di Lettere e Filosofia (19-22 Dicembre 1991), dir. Saverio Guida et Fortunata Latella, Messina, 1993, t. I, p. 273-308. Les autres Liederbücher sont : $h$ (Adam de la Halle), $r$ (Jehan de Renti) et $q$ (Comte de Bretagne).

${ }^{7}$ Les Grandes Chroniques de Saint-Denis, contenant l'histoire de France, depuis l'origine de la monarchie jusqu'à Charles $V$, et publiées d'après les manuscrits de la Bibliothèque Royale, avec notes et dissertations inédites par M. P. Paris, Paris, J. Techener, 1843, t. IV, p. 254-255.

${ }^{8}$ Voir Luca Barbieri, «Note sul Liederbuch di Thibaut de Champagne », Medioevo Romanzo, vol. 23, fasc. III, septembre-décembre 1999, p. 388-416.
} 
(« Amors me fet comencier», RS1268, L204-2), une chanson de croisade («Seignors sachiez», RS6, L204-49), une pastourelle (« J'aloie l'autrier errant », RS342, L204-27), une chanson d'amour (« En chantant vueil ma dolor descouvrir », RS1397, L204-25), une pièce de dialogue avec le dieu Amour («L'autre nuit en mon dormant», RS339, L204-32), avant de finir par des chansons d'amour et des jeux-partis.

«Chansonnier » est donc un appellatif commode pour des manuscrits extrêmement divers, posant de nombreux problèmes aux philologues qui entreprendraient de les classer.

\section{Problèmes philologiques}

Que le philologue ait pour objectif une édition «reconstructionniste » ou dite « bédiériste », il est nécessaire de pouvoir établir un stemma, c'est-à-dire un arbre généalogique de tous les manuscrits, afin d'obtenir une compréhension globale de la tradition manuscrite. Dans le domaine de la lyrique d'oïl, le classement suscite toujours des débats, depuis la hiérarchie proposée par Eduard Schwan en 1886 à la suite des travaux de Gröber sur les chansonniers de troubadours ${ }^{9}$.

Les chansonniers de trouvères (dont une vingtaine de complets) peuvent être regroupés en deux catégories en fonction de leur provenance, ou en trois familles (ou "archétypes ») : les chansonniers du nord de la France, picards (l'archétype nommé $\mathrm{s}^{\mathrm{I}}$ chez Schwan); les chansonniers de l'est de la France, lorrains, bourguignons voire franc-comtois (les archétypes $\mathrm{s}^{\mathrm{II}}$ et $\mathrm{s}^{\mathrm{III}}$ de Schwan). Les regroupements des différents manuscrits passent par l'identification de points communs, puis de fautes communes qui ne laissent place au doute, comme par exemple les omissions d'un seul vers qui sont partagées par plusieurs manuscrits. Dans

\footnotetext{
${ }^{9}$ Gustav Gröber, « Die Liedersammlungen der Troubadours », Romanische studien, 2, 1877, p. 337-670.
} 
les éditions de trouvères, on observe notamment que les chansonniers $\mathrm{M}, \mathrm{T}$, $A$, a et $Z$ ont des affinités au sein de l'archétype $s^{110}$. Les autres fautes communes permettent de grouper, pour $\mathrm{s}^{\mathrm{II}}, \mathrm{K}, \mathrm{N}, \mathrm{V}$ et $\mathrm{X}$, tandis que $\mathrm{O}$ est souvent indépendant par rapport au sous-groupe $\mathrm{KNVX}^{11}$, même si on manque fréquemment d'éléments solides pour les positions relatives de $\mathrm{K}$, $\mathrm{N}, \mathrm{V}$ et $\mathrm{X}$. Pour s ${ }^{\mathrm{III}}$, on arrive à prouver l'existence d'un groupe CU, rejoint parfois par I et $\mathrm{H}$.

Si la plupart des éditeurs lyriques reprennent le stemma général tracé par Schwan, certains font remarquer qu'il est difficile de prouver la distinction existant entre $s^{\text {II }}$ et $s^{\text {III }}$ car on n'arrive pas à opposer KNVX à $\mathrm{CU}^{12}$, d'autres évoquent la possibilité d'une source commune à $\mathrm{s}^{\mathrm{I}}$ et $\mathrm{s}^{\mathrm{III13}}$. L'éditeur de chansons de trouvères est ici comme bloqué ${ }^{14}$, car si le stemma général reste une hypothèse, l'établissement d'un stemma pour chaque pièce lyrique est souvent impossible en l'absence de fautes communes indiscutables ${ }^{15}$. Les divergences de leçons peuvent provenir de l'activité du

${ }^{10}$ Tracer un stemma fait souvent oublier les copies intermédiaires qu'il y a eues entre les divers chansonniers : on a ainsi peu de certitudes quant à la position relative des chansonniers $A, a$ et $Z$.

${ }^{11}$ Le flottement de $\mathrm{O}$, qui passe d'un archétype à un autre dans le stemma, semble indiquer qu'il est fortement contaminé.

${ }^{12}$ Philippe Ménard, «L'édition des textes lyriques du Moyen Âge, réflexions sur la tradition manuscrite de Guillaume le Vinier», dans Actes du III Congrès international de linguistique et philologie romanes, t. II, Québec, Les Presses de l'université Laval, 1976, p. 763-776.

${ }^{13}$ Luca Barbieri, «Contaminazioni, stratificazioni e ricerca dell'originale nella tradizione manoscritta dei trovieri ", dans La Tradizione della lirica nel medioevo romanzo. Problema di filologia formale. Atti del Convegno internazionale (FirenzeSiena, 12-14 novembre 2009), dir. L. Leonardi, Firenze, Edizioni del Galluzzo, 2011, p. 179-240.

14 À défaut d'un «arbre» que dessinent habituellement les éditeurs qui suivent le classement de Schwan, Luca Barbieri utilise des figures géométriques où chaque manuscrit est considéré comme un ensemble qui possède des affinités plus ou moins grandes avec un autre manuscrit. Voir Le Liriche di Hugues de Berzé, éd. Luca Barbieri, Milano, Cooperativa universitaria studio e lavoro, 2001.

${ }^{15}$ Harry H. Lucas, «L'édition des textes lyriques : schéma d'ensemble ou schéma individuel ? », dans Actes du $X^{e}$ congrès international de linguistique et de philologie romanes, Strasbourg 23-28 avril 1962, Paris, 1965, t. II, p. 697-703. L'auteur de l'article y rappelle à juste titre que Schwan n'a pas fait d'analyse exhaustive pour 
scribe lui-même, qui récite intérieurement ou de vive voix le texte qu'il copie après l'avoir $\mathrm{lu}^{16}$. Mais le problème majeur reste la contamination, c'est-à-dire la présence de plusieurs modèles : certaines variantes peuvent par exemple être proches d'un manuscrit, puis d'un autre situé dans une autre famille. Philippe Ménard remarque que dans le cas de Guillaume le Vinier, la pièce XXIX de son édition critique ${ }^{17}$ possède une rime fautive dans les manuscrits fréquemment sollicités par les éditeurs, dans la mesure où ils contiennent beaucoup de pièces, à savoir $\mathrm{KNVX}^{18}$,

Il serait donc téméraire de se lancer dans l'usage d'un seul manuscrit respecté scrupuleusement, comme le font les éditeurs bédiéristes à l'extrême, car on s'éloignerait alors du présumé original et de l'auteur au profit du copiste. Au contraire, la méthode dite «lachmanienne» ou «reconstructionniste» aura pour obstacle le faible nombre de fautes communes. Philippe Ménard propose une « voie moyenne », où il s'agit de publier un seul manuscrit, tout en corrigeant les leçons aberrantes, ou de choisir le meilleur manuscrit pour chaque poème. C'est alors le « goût» de l'éditeur qui permet de trancher entre les variantes, en évitant de transposer les graphies de toutes les pièces d'un trouvère en un seul dialecte, ce que l'on a pu faire jusqu'au milieu du $\mathrm{Xx}^{\mathrm{e}}$ siècle, et, ce qui est plus grave, sans en toucher un mot dans l'apparat critique. Le stemma est alors l'instrument qui doit servir à voir la hiérarchie des variantes et permet à terme de faire les corrections jugées les meilleures.

Dans la mesure où les chansonniers de trouvères notent plus souvent les mélodies que les chansonniers occitans, on a pu penser que le critère

\footnotetext{
établir ses classements de manuscrits.

${ }^{16}$ Keith Busby, «Mise en texte as Indicator of Oral Performance in Old French Verse Narrative ", dans Performing Medieval Narrative, dir. Evelyn Birge Vitz, Nancy Freeman Regalado et Marilyn Lawrence, Cambridge, 2005, p. 61-71.

${ }^{17}$ Les Poésies de Guillaume le Vinier, éd. Philippe Ménard, Genève, Droz, 1983.

${ }^{18}$ Philippe Ménard, «L'édition des textes lyriques du Moyen Âge... », art. cit., p. 769.
} 
musical permettrait de classer les manuscrits. Les recherches des musicologues tendent cependant à montrer que l'on ne peut pas tirer de conclusions définitives par le regroupement des mélodies, qui n'a parfois rien à voir avec le regroupement par textes ${ }^{19}$. Hendrik Van der Werf soutient que des transpositions auraient été faites par des vocalistes faisant évoluer le matériau mélodique ${ }^{20}$.

Les chansonniers méritent d'être mieux connus dans leur singularité, et c'est ce à quoi s'est attelé depuis la fin des années 90 le projet international Intavulare qui a pour but de produire les tables de tous les chansonniers $^{21}$, aidant le philologue à mieux saisir l'organisation de ces recueils qui, souvent, ne peuvent être lus tels quels, dans la mesure où se sont succédé insertions, suppressions et déplacements. L'édition critique des trouvères a donc tout à gagner de la redécouverte de ces chansonniers qui sont moins bien connus que leurs équivalents occitans.

${ }^{19}$ Voir Gérard Le Vot, « Les transpositions/transformations mélodiques dans la monodie des trouvères. Un problème d'analyse ", dans Histoire, humanisme et hymnologie: mélanges offerts au professeur Édith Weber, Paris, Presses de l'Université Paris-Sorbonne, 1997, p. 149-162.

${ }^{20}$ Hendryk Van der Werf, The Chansons of the troubadours and trouvères. A study of the melodies and their relation to the poems, Utrecht, Oosthoek, 1972, p. 26-30.

${ }^{21}$ Les tables concernant les chansonniers provençaux ont été publiées parallèlement. Les chansonniers français qui ont fait l'objet d'une publication dans la série Intavulare sont A (Arras, Bibliothèque Municipale, 657), C (Berne, Stadtbibliothek, 389), H (Modène, Biblioteca Nazionale Estense, Estero 45, Alpha R.4.4), U (Paris, BnF, fr. 20050), Z (Sienne, Biblioteca Comunale, H.X.36), a (Rome, Biblioteca Apostolica Vaticana, Regina 1490) et b (Rome, Biblioteca Apostolica Vaticana, Regina 1522). 